DAMTP-R/95/4

gr-qc/9502017

January, 1995

\title{
Quantum Coherence and Closed Timelike Curves
}

\author{
S. W. Hawking \\ Department of Applied Mathematics and Theoretical Physics \\ University of Cambridge \\ Silver Street \\ Cambridge CB3 9EW \\ UK
}

\begin{abstract}
Various calculations of the $S$ matrix have shown that it seems to be non unitary for interacting fields when there are closed timelike curves. It is argued that this is because there is loss of quantum coherence caused by the fact that part of the quantum state circulates on the closed timelike curves and is not measured at infinity. A prescription is given for calculating the superscattering matrix $\$$ on space times whose parameters can be analytically continued to obtain a Euclidean metric. It is illustrated by a discussion of a spacetime in with two disks in flat space are identified. If the disks have an imaginary time separation, this corresponds to a heat bath. An external field interacting with the heat bath will lose quantum coherence. One can then analytically continue to an almost real separation of the disks. This will give closed timelike curves but one will still get loss
\end{abstract}


of quantum coherence. 


\section{Introduction}

This paper is about what sense, if any, can be made of quantum field theory on a spacetime background that contains closed timelike curves. The development of causality violation in a bounded region is classically forbidden in the sense that it can not occur if the weak energy condition holds [1]. However, quantum field theory in curved spacetime has many examples like the Casimir effect where the expectation value of the energy momentum tensor fails to obey the weak energy condition. It has therefore been suggested [2] that an advanced civilization might be able to create a wormhole in spacetime which could be used to travel into the past. This has led to a lot of interest in the problem of the formulation and behavior of quantum field theory in spacetimes with closed timelike curves.

In general, it seems that divergences in the energy momentum tensor occur when one has closed or self intersecting null geodesics [3]. These divergences may create spacetime singularities which prevent one from traveling through to the region of closed timelike curves [1]. However, Kim and Thorne [3] have suggested that quantum gravitational effects may smear out the divergences and lead to a non singular spacetime. It is therefore of interest to consider the properties of quantum field theory in spacetimes with closed timelike curves.

In particular, a number of authors have studied what I shall call, confined causality violating spacetimes. In these, there are well behaved initial and final regions and the causality violations are restricted to a region in the middle. One can make this definition more precise but I shall refrain from doing so in order that I can discuss as wide a class of examples as possible. On such spacetimes, one might hope to calculate an $S$ matrix which would relate the quantum state in the final region to the state in the initial region. Some authors have claimed $[4,5]$ that this $S$ matrix will be unitary for free fields but will be non unitary if there are interactions. To try to make sense of such non unitarity, Hartle [6] has suggested that the usual amplitudes should be normalised by a factor that depends on the initial state. This would restore conservation of probability but at the heavy price of making quantum mechanics non linear. In principle, one would be able detect the non linearity produced by a wormhole, which an advanced civilization might create in the distant future. There would be a paradox, if this information were to cause the advanced civilization to change its mind about creating the wormhole. But such paradoxes occur anyway with closed timelike curves.

Anderson [7] has suggested that one should evolve the quantum state only with the unitary part of the $S$ matrix $U=\left(S S^{\dagger}\right)^{-1 / 2} S$. The trouble with this proposal is that it doesn't obey the usual composition law [8]: the unitary part of $S_{1} S_{2}$ is not the product

of the unitary part of $S_{1}$ and $S_{2}$ separately. A third proposal is to extend the $S$ to be a 
unitary transformation on a larger Hilbert space [8]. The trouble with this idea is that the larger Hilbert space may have to have an indefinite metric.

The message of this paper is that there is no need to propose non linear modifications of quantum theory, or indefinate metrics on Hilbert space. The reason that the $S$ matrix, calculated according to the usual rules, is non unitary, is that there is loss of quantum coherence when there are closed timelike curves. This means that the probability to go from an initial state to a final state is given by a superscattering operator, $\$$, rather than by $S S^{\dagger}$. Thus it does not matter that the object that one might think was the $S$ matrix, is not unitary.

A proposal has been made by Deutsch [9] and Politzer [10] for calculating the evolution in the presence of closed timelike curves. This approach is based on finding a consistent solution for the density matrix. This solution will involve loss of quantum coherence in general. However, it will also depend in a non linear way on the initial state [11], which means that one loses the superposition principle.

If one simply requires that the quantum theory is linear, the most general relation between the initial and final situations is not an $S$ matrix but a superscattering operator, $\$$, that maps initial density matrices, to final ones. In what follows it will be helpful to use index notation. I shall represent a vector in a Hilbert space, by a quantity with an upper index.

$$
\lambda^{A} \in \mathcal{H}
$$

The corresponding vector in the complex conjugate Hilbert space, will carry a lower index.

$$
\bar{\lambda}_{A} \in \overline{\mathcal{H}}
$$

The $S$ matrix is a linear map from the initial Hilbert space to the final Hilbert space, so it can be written as a two index tensor.

$$
\psi_{+}{ }^{A}=S^{A}{ }_{B} \psi_{-}{ }^{B}
$$

However, the most general description of the quantum state of a system is not a vector in a Hilbert space, but a density matrix. This can be regarded as a Hermitian two index tensor on Hilbert space. Then the most general linear evolution is given by a four index tensor that maps initial density matrices to final ones.

$$
\rho_{+B}^{A}=\$_{B C}^{A}{ }^{D} \rho_{-}{ }^{C}{ }_{D}
$$

Many familiar quantum systems obey the Axiom of Asymptotic completeness [12]. This requires that the Hilbert space of the interaction region in the middle is isomorphic 
to the initial and final Hilbert spaces. In other words, there are unitary maps between the interaction region Hilbert space and the initial and final Hilbert spaces. If this is the case, there will evidently be a unitary map from the initial Hilbert space to the final Hilbert space. The superscattering operator $\$$ will factorize into the product of an $S$ matrix and its adjoint.

$$
\$_{B C}{ }^{D}=S_{C}^{A} \bar{S}_{B}^{D}
$$

In this situation, a density matrix corresponding to a pure quantum state will be carried into a pure quantum state. There will be no loss of quantum coherence.

However, there are quantum systems that do not obey the Axiom of Asymptotic Completeness. An example is provided by a particle interacting with a heat bath. A heat bath is not in a single quantum state. Rather it can be in any quantum state $|n\rangle$ with probability $\exp -\frac{E_{n}}{T}$ In other words, it is in a mixed quantum state. A particle that is initially in a pure quantum state, which interacts with the heat bath, will end up in a mixed quantum state. This loss of quantum coherence is to be expected: information about the original quantum state of the particle is lost into the heat bath. However, I will give examples of systems with closed timelike curves that are very similar to particles interacting with a heat bath. The only difference is that the temperature of the heat bath is imaginary. This corresponds to a spacetime that is identified periodically in real Lorentzian time, rather than periodically in imaginary time, as in a normal heat bath. It should therefore come as no surprise that one gets loss of quantum coherence in these cases. More generally, whenever one has confined causality violations, one has part of the quantum state that is circulating on closed timelike curves. When one makes measurements at infinity, one does not see this part of the state. One will therefore have to describe the state at infinity by a mixed state, obtained by tracing out over the part of the state that one can't see.

In this paper I shall show that the usual rules of quantum theory seem to lead to loss of quantum coherence when there are closed timelike curves. I should emphasize that this loss is not an optional feature that one can choose whether or not to have in the theory. Rather, like radiation from black holes, it is an inevitable consequence of the standard assumptions of quantum field theory in curved spacetime. The only way to protect the purity of quantum states is either to abandon one or more of these standard assumptions, or subscribe to the Chronology Protection Conjecture [1]. This says that quantum effects become so large when closed timelike curves are about to appear that they either prevent the curves appearing or they bring the spacetime to an end at a singularity. In either case the laws of physics conspire to prevent causality violations. 


\section{Euclidean Approach}

It is clear how to define quantum field theory on a curved spacetime background that is globally hyperbolic. That is to say, it can be covered by a family of Cauchy surfaces. In this case, one can choose the commutator of two free field operators to be the half advanced minus half retarded Green function, which is well defined.

$$
[\phi(x), \phi(y)]=i D(x, y)
$$

However, it is much less clear how to proceed if the spacetime is not globally hyperbolic, and in particular, if it contains closed timelike curves. In this case, it is not clear how to generalize the half advanced minus half retarded Green function and field operators at points that are locally spatially separated may not commute because the points can be joined by a timelike curve that goes round a large loop and returns to the same neighbourhood.

The approach I shall adopt is to analytically continue the parameters of the spacetime with closed timelike curves to get a metric with a real Euclidean section. On this section, all the field operators commute and the Green functions are well defined. One then analytically continues back, both in the parameters of the metric, and in the points themselves in a certain order, to get Green functions in the original Lorentzian spacetime. One can then calculate the superscattering operator from the Green fun ctions according to certain rules which involve displacing the points slightly into the complex. This is equivalent to the usual $i \epsilon$ prescription.

One can illustrate this with a simple causality violating spacetime which has been discussed by Politzer [8]. Two flat spacelike three dimensional disks of radius $R$ are located

in Minkowski space at $t=-\frac{1}{2} T$ and $t=\frac{1}{2} T$ and the same spatial coordinates. One makes the following identifications:

1 The lower surface of the bottom disk is identified with the upper surface of the top disk.

2 The upper surface of the bottom disk is identified with the lower surface of the top disk.

The resultant spacetime is geodesically incomplete at the edges of the disks. However, one can impose boundary conditions there that make the wave equation well behaved, at any rate locally. The first identification is not very significant and is imposed just to avoid free surfaces. But the second introduces closed timelike curves in the region between the disks.

In order to define the Green functions, I shall first take the separation between the disks $T$ to be pure imaginary. One then has a Euclidean spacetime with time coordinate 
$\tau=i t$. On this one can define Green functions in the normal way as $n$ point expectation values.

$$
\begin{gathered}
G\left(x_{1}, x_{2}, \ldots, x_{n}\right)=\left\langle\phi\left(x_{1}\right) \phi\left(x_{2}\right) \ldots \phi\left(x_{n}\right)\right\rangle \\
=\int d[\phi] \phi\left(x_{1}\right) \phi\left(x_{2}\right) \ldots \phi\left(x_{n}\right) e^{-I}
\end{gathered}
$$

In the case of free fields, the $n$ point function will be built out of all combinations of two point functions. But if there are interactions, one will have the usual Feynman diagram expansion in terms of the two point function on the background.

On the Euclidean spacetime, all points are spacelike separated from each other. This means that the field operators at different points commute with each other. Thus the $n$ point Green function does not depend on the order in which the $n$ points are taken, as is obvious from the representation of the Green function by a path integral. On the other hand, the $n$ point Wightman functions in Lorentzian spacetime certainly do depend on the order of the points, because the field operators do not commute at timelike separated points. The way this comes about is that one analytically continues the $n$ point expectation values from the Euclidean to the Lorentzian regime, keeping a small imaginary time displacement between each field point [12]. The displacement is in the positive imaginary time direction between each point in the Lorentzian expectation value reading from left to right. That is, for the expectation value

$$
\left\langle\phi\left(x_{1}\right) \phi\left(x_{2}\right) \ldots \phi\left(x_{n}\right)\right\rangle
$$

one requires that $\operatorname{Im}\left(x_{1}^{0}\right)<\operatorname{Im}\left(x_{2}^{0}\right)<\ldots<\operatorname{Im}\left(x_{n}^{0}\right)$. The purpose of the displacement is to evaluate the analytically continued expectation values on the right side of the singularities that occur on the complex light cone. It is equivalent to the usual $i \epsilon$ prescription, for integrating round the singularities in the propagator. 


\section{The Superscattering Operator $\$$}

I now come to the rules for calculating the superscattering matrix from the Lorentzian expectation values [12]. One makes the usual assumption, that the field in the initial and final regions can be expanded in terms of annihilation and creation operators.

$$
\phi=\sum_{i}\left\{f_{i} a_{i}+\bar{f}_{i} a_{i}^{\dagger}\right\}
$$

The annihilation operators $a_{i}$ are multiplied by positive frequency wave functions $f_{i}$ and the creation operators $a^{\dagger}$ are multiplied by negative frequency wave functions $\bar{f}_{i}$. One can invert this relation and express the annihilation and creation operators as integrals over spacelike surfaces of the field operator $\phi$ with negative or positive frequency wave functions.

$$
a_{i}=\int_{\Sigma} \bar{f}_{i}(x) \overleftrightarrow{\nabla}_{\mu} \phi(x) d \Sigma^{\mu}(x)
$$

One is interested in the expectation value of certain operators $Q$ in a given initial state with density matrix $\left|\psi_{-}\right\rangle\left\langle\psi_{-}\right|$. This will be given by

$$
\left\langle I^{\dagger} Q I\right\rangle
$$

where $I$ is a string of creation operators that create the given initial state:

$$
\left|\psi_{-}\right\rangle=I\left|0_{-}\right\rangle
$$

In particular, one is interested in the probability of the final state containing a set of particles created by the string $F$ of creation operators. This would correspond to taking $Q=F F^{\dagger}$. Thus the superscattering matrix element between the initial state $\left|\psi_{-}\right\rangle\left\langle\psi_{-}\right|$ and the final state $\left|\psi_{+}\right\rangle\left\langle\psi_{+}\right|$is determined by $\left\langle I^{\dagger} F F^{\dagger} I\right\rangle$.

One can express the annihilation and creation operators in terms of the field operators. In this way, the superscattering matrix can be calculated from an integral of expectation values with initial and final wave functions. In order to get the right operator ordering in the expectation value, these integrals over the initial and final surfaces have to be slightly displaced in the imaginary time direction. The rule is, the initial creation operators have the greatest displacement in the positive imaginary time direction. They are followed by the final annihilation operators, the final creation operators, and then the initial annihilation operators. This is illustrated in Figure 1.

In Minkowski space positive frequencies propagate only towards the negative imaginary time direction, and negative frequencies propagate only towards positive imaginary 
time. This means that the data from the string $F^{\dagger}$ that corresponds to the annihilation operators for the final state can propagate only upwards in imaginary time. Because the final state annihilation operators act on a space like surface slightly above the real time axis, the only surface they can propagate to is the surface on which the initial state creation operators act. Similarly, the positive frequency data from the final state creation operators can only propagate downwards, and the only surface it can reach is that on which the initial state annihilation operators act. Thus in this case, the diagram that represents going from initial state $\left|\psi_{-}\right\rangle\left\langle\psi_{-}\right|$to the final state $\left|\psi_{+}\right\rangle\left\langle\psi_{+}\right|$falls into two disconnected parts. This means that the probability for going from initial to final factors into an $S$ matrix, corresponding to the upper part of the diagram, and its adjoint, corresponding to the lower part. In this situation, there is no loss of quantum coherence and the $S$ matrix is unitary.

Suppose however one identifies disks in Minkowski space at $\pm T$ where $T$ is imaginary. Then negative frequency data from the final state annihilation operators will be able to propagate upwards in imaginary time to the upper disk and re-emerge at the lower disk. From there it can propagate upwards to the final state creation operators, rather than the initial state creation operators. Similarly, the data from the initial state creation operators can propagate downwards to the initial state annihilation operators. In this way, one gets a diagram that is not divided into two parts by the real time axis. This means that the probability will not factor into an $S$ matrix and its adjoint, and there will be loss of quantum coherence. Physically, this is what one would expect. The region between the disks is identified periodically in imaginary time. Thus it corresponds to a heat bath and will be in a mixed quantum state. A free field would propagate straight through the heat bath and not notice its existence but an interacting field will be affected and will lose quantum coherence to the heat bath.

In the two disk example that has been considered, the expectation value of $F F^{\dagger}$ will be non zero even if the string $I$ of initial creation operators is empty. This means one can detect particles in the final state even when there were none present originally. The reason one gets such energy non conservation is that one is considering field theory on a fixed background that is not time translation invariant. One would expect energy conservation only in a full quantum theory of gravity in which one summed over all metrics as well as all fields in those metrics. Nevertheless, in the context of quantum field theory in curved backgrounds, it may make sense to consider the change in the final state brought about by the application of the initial state creation and annihilation operators. Thus one should calculate the superscattering matrix not from

$$
\left\langle I^{\dagger} F F^{\dagger} I\right\rangle
$$


But from

$$
\left\langle I^{\dagger} F F^{\dagger} I\right\rangle-\left\langle F F^{\dagger}\right\rangle
$$

The idea is now to rotate the separation of the disks from imaginary to almost real. One should keep a small imaginary part to the separation. This damps possible divergences from high frequency modes by reducing them by a thermal factor, $\exp (-E / T)$, with $1 / T$ imaginary but with a small positive real part. Keeping a small imaginary part also means that one can rotate the separation of the disks from imaginary to almost real time without encountering any singularities. If one didn't keep a small imaginary part to the separation, one couldn't use analytical continuation from a Euclidean spacetime, to determine the Green function, but would have to find some other prescription.

There is a question of which direction one should rotate the separation from imaginary time to almost real. In a path integral over metrics, presumably both directions will occur. I therefore think one should take the sum of the rotations in both directions. This will ensure that the probabilities of going from initial to final are real.

As long as one keeps a small imaginary part to the separation, one will lose quantum coherence, like in the pure imaginary separation case. Thus it seems that there will be loss of quantum coherence in the Lorentzian case. Physically this is reasonable, because one has external fields interacting with a heat bath at an imaginary temperature with a small real part. More generally, one might expect loss of quantum coherence whenever one has closed timelike curves, because there will be a part of the quantum state that one doesn't measure initially or finally.

The superscattering matrix for this spacetime will not conserve energy because one has been considering quantum field theory on a fixed background and not taking back reaction into account. In two dimensional black hole calculations, where we know how to include back reaction, one finds that the superscattering matrix conserves energy [13]. 


\section{Conclusion}

I have shown that the reason the usual rules seem to lead to an $S$ matrix that is non unitary is that there is part of the quantum state that circulates on the closed timelike curves and is not measured at infinity. This leads to loss of quantum coherence and a superscattering matrix that does not factor. Thus if one multiplies the object that would normally be the $S$ matrix by its adjoint, one does not get the probability for going from the initial, to the final state. This means there is no reason for the $S$ matrix to be unitary.

My approach to calculating the superscattering operator in the presence of closed timelike curves, consisted of two elements. The first was a set of rules that give the superscattering matrix in terms of the ordered Lorentzian expectation values, analytically continued to a neighbourhood of the real time axis. These rules are not a matter of choice. They are forced on us by the usual assumptions of quantum field theory on a fixed background. The second element of my approach, was to analytically continue the parameters of the Lorentzian metric, to obtain a Euclidean one. One could uniquely define the Green functions in this metric to be the $n$ point expectation values. One could then analytically continue in both the field points and the parameters of the solution to get the Lorentzian expectation values, which could then be used to calculate the superscattering matrix. There might be alternative, inequivalent ways of defining the Lorentzian expectation values but for the reasons I have given, I think that any reasonable alternative would also give loss of quantum coherence.

Spacetimes with closed timelike curves show that loss of quantum coherence is not confined to black holes, but can occur with other spacetimes with non trivial causal structure. This is important, because some people have claimed that the Planck scale physics at the end of black hole evaporation, will restore quantum coherence. However, in a causality violating spacetime, the curvature could be small everywhere and Planck scale physics would not come in. This reinforces my conviction that quantum coherence really is lost in black hole evaporation.

Personally, I don't believe that closed timelike curves will occur, at least on a macroscopic scale. I think that the Chronology Protection Conjecture will hold and that divergences in the energy momentum tensor will create singularities before closed time like curves appear. However, if quantum gravitational effects somehow cut off these divergences, I'm quite sure that quantum field theory on such a background will show loss of quantum coherence. So even if people come back from the future, we won't be able to predict what they will do. 


\section{Acknowledgements}

I am grateful to Mike Cassidy, Jim Hartle and David Politzer for discussions.

\section{References}

[1] S. W. Hawking, Phys. Rev. D46, 603 (1992)

[2] M. S. Morris, K. S. Thorne and U. Yurtsever, Phys. Rev. Lett. 61, 1446 (1988)

[3] S-W. Kim and K. S. Thorne, Phys. Rev. D43, 3929 (1991)

[4] D. G. Boulware, Phys. Rev. D46, 4421 (1992)

[5] J. L. Friedman, N. J. Papastamatiou and J. Z. Simon, Phys. Rev. D46, 4456 (1992)

[6] J. B. Hartle, Phys. Rev. D49, 6543 (1994)

[7] A. Anderson, gr-qc 9405058 (1994)

[8] C. J. Fewster and C. G. Wells, hep-th 9409156 (1994)

[9] D. Deutsch, Phys. Rev. D44, 3197 (1990)

[10] H. D. Politzer, Phys. Rev. D49, 3981 (1994)

[11] M. J. Cassidy, gr-qc 9409003, to be published (1994)

[12] S. W. Hawking, Commun. Math. Phys. 87, 395 (1982)

[13] S. W. Hawking, Phys. Rev. D50, 3982 (1994) 